\title{
Measurement of Narcolepsy Symptoms: the Narcolepsy Severity Scale
}

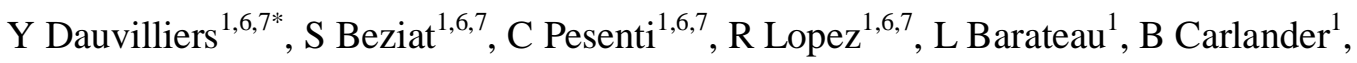

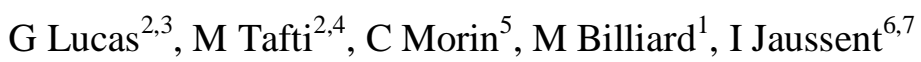

${ }^{1}$ CHU Montpellier, Hôpital Gui-de-Chauliac, Service de Neurologie, Unité du Sommeil, Centre National de Référence pour la Narcolepsie, Montpellier, France

${ }^{2}$ Center for Integrative Genomics, University of Lausanne, Lausanne, Switzerland

${ }^{3}$ Centre Neuchâtelois de Psychiatrie, Neuchâtel, Switzerland

${ }^{4}$ Center for Investigation and Research in Sleep, Lausanne University Hospital, Lausanne, Switzerland.

${ }^{5}$ Department of Psychology, Université Laval, Quebec City, Quebec, Canada.

${ }^{6}$ Inserm, U1061, Montpellier, France

${ }^{7}$ Université Montpellier, Montpellier, France

* Corresponding author:

Yves Dauvilliers, MD, $\mathrm{PhD}$

Service de Neurologie, Hôpital Gui-de-Chauliac, 80 avenue Augustin Fliche, 34295 Montpellier cedex 5, France

Phone: (33) 467337277

Fax: (33) 467337285

Email: ydauvilliers@yahoo.fr

\section{Running title: Narcolepsy Severity Scale}

Words: 3201

Abstract: 226

References: 27

Tables: 3

\section{Figure 2, suppl figure: 1}

Appendix A: the English and French versions of the Narcolepsy Severity Scale

Key words: narcolepsy, scale, sleepiness, cataplexy, severity, questionnaire 
Neurology search terms: [ 245 ]

\section{AUTHOR CONTRIBUTIONS:}

Y Dauvilliers: drafting/revising the manuscript for content, including medical writing for content; study concept or design; interpretation of data analysis, study supervision and coordination.

I Jaussent: drafting/revising the manuscript for content, statistical analysis and interpretation of data

S Beziat and G Lucas : revising the manuscript for content; statistical analysis and interpretation of data analysis.

C Pesenti, R Lopez, L Barateau, B Carlander, M Tafti, C Morin, M Billiard : acquisition of data and revising the manuscript for content

\section{ACKNOWLEDGMENT:}

STUDY FUNDING: This was not an industry-supported study.

\section{Conflicts of interest:}

We declare no conflicts of interest related to this article.

Y Dauvilliers received funds for seminars, board engagements and travel to conferences by UCB Pharma, Jazz, Theranexus, Flamel and Bioprojet.

M Tafti received funds for seminars and board engagements and travel to conferences by UCB Pharma and GSK.

C Morin received research support from Novartis and served as consultant for Merck

RL has received funds for speaking with UCB Pharma and Shire

S Beziat, L Barateau, B Carlander, C Pesenti, G Lucas, M Billiard and I. Jaussent have no disclosure. 


\section{Abstract}

Objective: Validation of the Narcolepsy Severity Scale (NSS), a brief clinical instrument to evaluate the severity and consequences of symptoms in patients with narcolepsy type 1 (NT1).

Methods: A 15-item scale to assess the frequency and severity of excessive daytime sleepiness, cataplexy, hypnagogic hallucinations, sleep paralysis and disrupted nighttime sleep was developed and validated by sleep experts with patients' feedback. Seventy untreated and 146 treated adult patients with NT1 were evaluated and completed the NSS in a single reference sleep center. The NSS psychometric properties, score changes with treatment and convergent validity with other clinical parameters were assessed.

Results: The NSS showed good psychometric properties with significant item-total score correlations. The factor analysis indicated a three-factor solution with good reliability, expressed by satisfactory Cronbach's $\alpha$ values. The NSS total score temporal stability was good. Significant NSS score differences were observed between untreated and treated patients (dependent sample: 41 patients before and after sleep therapy; independent sample: 29 drugfree and 105 treated patients). Scores were lower in the treated populations (10-point difference between groups), without ceiling effect. Significant correlations were found between NSS total score and daytime sleepiness (Epworth Sleepiness Scale, Mean Sleep Latency Test), depressive symptoms and health-related quality of life.

Conclusions: The NSS can be considered a reliable and valid clinical tool for the quantification of narcolepsy symptoms to monitor and optimize narcolepsy management.

\section{INTRODUCTION}


Narcolepsy is a rare, disabling chronic neurologic disorder that is classically characterized by four major symptoms: excessive daytime sleepiness (EDS), cataplexy, hypnagogic hallucinations and sleep paralysis. ${ }^{1,2}$ Disrupted nighttime sleep is also frequent and should be considered as the fifth narcolepsy symptom. Following the breakthrough discovery of hypocretin deficiency, narcolepsy with cataplexy was recently renamed Narcolepsy Type 1 (NT1, or hypocretin-deficiency syndrome), according to the third edition of the International Classification of Sleep Disorders. ${ }^{3}$

EDS is the most common presenting symptom and the most disabling one and is required for the diagnosis. Cataplexy is the most specific symptom of NT1 and is defined as a sudden, transient partial or total loss of muscle tone triggered by strong emotions. ${ }^{4}$ Cataplexy frequency and intensity vary among patients. Half of them may experience both partial and complete attacks, whereas other patients have only partial cataplexy. ${ }^{1,4}$ The estimated prevalence of hypnagogic hallucinations ranges from $30 \%$ to $80 \%$ of patients and that of sleep paralysis from $25 \%$ to $50 \%{ }^{4,5}$ Disrupted nighttime sleep affects $22 \%$ to $45 \%$ of patients. ${ }^{5}$ Only a minority of patients (less than 25\%) suffers from all five symptoms, with large variability in terms of symptom frequency, intensity and effect on daily life. ${ }^{1,2,6}$ Although narcolepsy is associated with substantial morbidity and impaired health-related quality of life, the severity and consequences of each of these symptoms on the disease burden remain unclear. $^{7-10}$

In recent years, a number of drugs have been developed to efficiently treat narcolepsy. However, there is a lack of narcolepsy-specific instruments to monitor symptom severity and their changes following treatment. A variety of subjective (e.g., Epworth sleepiness scale, ESS) and objective (e.g., Maintenance of Wakefulness Test, MWT) tests have been used to evaluate the severity of daytime sleepiness and its response to treatment. ${ }^{11,12}$ However, these tools focus only on EDS and their scores may not necessarily reflect the patient's 
functionality or symptomatic complaints. Therefore, the other narcolepsy symptoms (cataplexy, hypnagogic hallucinations, sleep paralysis and disrupted nighttime sleep) are often not properly monitored.

To overcome this limitation, we developed a brief, self-report instrument that we called Narcolepsy Severity Scale (NSS) to measure the severity of the five main narcolepsy symptoms. The aim of this study was to assess NSS psychometric properties, its validity, reliability and responsiveness to narcolepsy medications in a well-defined population of adult patients with NT1.

\section{METHODS}

\section{NSS development}

The domains to be covered by the NSS were first defined by sleep experts (YD, BC, MT, CM, MB) on the basis of the main NT1 symptoms and their consequences. Several preliminary versions (for item selection and reduction) were developed and pilot-tested by physicians and few patients from the France-Narcolepsy Patients Association. The final set of 15 items retained for the NSS assesses the severity, frequency and effect of the five key narcolepsy symptoms (EDS: seven items; cataplexy: three items; hallucinations: two items; sleep paralysis: two items; disturbed nighttime sleep: one item) with good readability and comprehensiveness by the target population. The six items that assess symptoms frequency are rated using a six-point Likert scale, while the nine items that describe the symptom effect on daily life are rated using a four-point Likert scale. Their sum gives a total score that ranges from 0 to 57; higher scores indicate more severe and frequent symptoms. Patients are asked to rate the frequency, severity and consequences of each symptom based on their last month experience. About five minutes are needed, on average, to complete the NSS. The original 
French NSS questionnaire was translated into English for the readers of this article (Appendix A).

\section{Patients}

For this study, 175 consecutive adult patients with NT1 (71 women and 104 men; mean age 41.50 \pm 17.36 years) were recruited from the Reference National Center for Narcolepsy of Montpellier, France. NT1 was diagnosed following the ICSD-3 criteria: history of clear-cut cataplexy and mean sleep latency on the Multiple Sleep Latency Test (MSLT) $\leq 8$ minutes with $\geq 2$ sleep onset REM periods (SOREMPs) and cerebrospinal fluid (CSF) hypocretin-1 level $<110 \mathrm{pg} / \mathrm{ml}$ (84 patients had a lumbar puncture and all had CSF hypocretin-1 levels lower than $110 \mathrm{pg} / \mathrm{ml}){ }^{3}$ All patients had the HLA DQB1*06:02 genotype.

\section{Procedure}

The NSS was completed by the 175 consecutive patients (70 patients were untreated and 105 were taking medications to treat EDS or cataplexy). In the untreated group, 41 patients (18 females and 23 males, mean age $38.20 \pm 13.84$ years) completed the NSS a second time after treatment with stimulant and/or anticataplectic drugs (median interval: 11 months, range: 1-87 months) (Figure 1). Therefore, the independent sample included 29 untreated and 105 treated patients who completed the NSS at the first visit, while the dependent sample included 41 patients that completed the NSS twice (first as untreated and then as treated patients).

\section{Measures}

A standardized clinical evaluation was performed at the time of the study by a sleep expert physician that included questions related to: 1) demographic characteristics, 2) educational level ( $<12$ years $/ \geq 12$ years of education); 3 ) body mass index (BMI) (dichotomized in: nonobese $\left[<30 \mathrm{~kg} / \mathrm{m}^{2}\right]$ and obese $\left.[\geq 30]\right) ; 4$ ) diagnosis delay (i.e., delay between the onset of 
narcolepsy symptoms and diagnosis of NT1); 5) severity of depressive symptoms assessed by the Beck Depression Inventory II (BDI-II), categorized as "no-mild" (BDI-II score: $\leq 19$ ) and "moderate-severe" depressive symptoms (BDI-II score: 20-63) ${ }^{13}$; 6) EDS presence and severity, assessed by the ESS and categorized as no EDS (ESS score: $\leq 10$ ), mild EDS (ESS score: $11-15)$ and severe EDS (ESS score $\geq 16)^{11}$; 7) the presence of disturbed nighttime sleep and its severity evaluated with the Insomnia Severity Index (ISI) and classified as no insomnia (ISI score: $\leq 7$ ), sub-threshold insomnia ( score: 8 -14), moderate to severe insomnia $(\text { score }>14)^{14}$; and 8) quality of life assessed with the European Quality of Life instrument that includes a health self-classification system with five dimensions (mobility, self-care, usual activities, pain and anxiety/depression), each with three response levels (no problem, some problems, severe problems) (EQ-5D utility values) and a visual analog scale (EQ-VAS). ${ }^{15}$

Data on total sleep time, sleep efficiency on the polysomnography, mean sleep latency and number of SOREMPs on the MSLT were recorded in drug-free patients, and the mean sleep latency on the MWT in treated patients. The use of drugs to treat EDS (modafinil, methylphenidate, pitolisant, mazindol and sodium oxybate) and cataplexy (antidepressants and sodium oxybate) was reported for each patient at the time of the evaluation.

This study was approved by the institutional review board of the University of Montpellier, France.

\section{Statistical analysis}

The demographic characteristics and clinical data were described using means and standard deviations for continuous variables and percentages or frequencies for categorical variables. The independent Student's $t$-test was used to compare continuous variables between treated and untreated patients (independent groups), and the Chi-square or Fisher's exact tests for categorical variables. Associations between continuous variables were assessed using the 
Pearson correlation coefficient. The dependent $t$-test was used to compare differences between continuous variable at two different time points or two different conditions and the Mc Nemar's test for paired categorical data.

To analyze NSS factor structure, a principal components factor analysis was conducted using the data of the subjects who completed the NSS questionnaire at the first visit using a varimax rotation. The number of factors was determined on the basis of the obtained factor loadings and eigenvalues. Sampling adequacy was measured by calculating the Kaiser-Meyer-Olkin (KMO) index. The internal consistency (reliability) of the scores for the different items was estimated using the Cronbach's coefficient $\alpha$.

Receiver-operating characteristic (ROC) curves were drawn using the NSS total scores to identify the cut-off that best predicted the group with efficient treatment. The best cut-off was defined as the point with the highest Youden Index [(specificity + sensibility) -1$]$.

Statistical significance was set at $\mathrm{p}<0.05$. Statistical analyses were performed using SAS version 9.4 and the Stata 11 software (StataCorp 2007; Stata Statistical Software: Release 11. College Station, TX: StataCorp LP).

\section{RESULTS}

\section{Population}

Seventy consecutive untreated ( 29 females and 41 males, mean age $40.46 \pm 15.77$ years) and 105 consecutive treated adult patients (42 females and 63 males, mean age 42.20 \pm 18.39 years) with a diagnosis of NT1 completed the NSS at the first evaluation. Their sociodemographic, clinical and polysomnographic features are reported in Table 1.

Among the 105 treated patients of the independent sample, 63 were taking both stimulant 
(modafinil, methylphenidate, pitolisant, mazindol) and anticataplectic drugs (sodium oxybate, venlafaxine, duloxetine, clomipramine), 40 patients only a stimulant, one patient venlafaxine and one patient sodium oxybate. Among the 41 patients of the dependent sample, 24 took both stimulant and anticataplectic drugs, 16 only a stimulant, and one venlafaxine.

\section{Construct validity}

\section{Item convergent validity}

The correlations between individual items and the total score of the NSS were always significant and positive in the whole sample ( $\mathrm{n}=175$ patients) and in the treated and drug-free groups (independent sample). The correlations of the individual items with the total score were equal or greater than 0.50 [range: $0.50-0.72$ ] in the whole sample, 0.44 [range: $0.44-$ 0.66 ] in the treated group, and 0.41 [range: $0.41-0.75$ ] in the non-treated group. The only exception concerned the item 5 score (“How do you feel generally after one sleep attack?") that had a correlation of 0.30 in the whole sample, of 0.19 in the non-treated group and of 0.34 in the treated group. However, due to the clinical importance of this item, we choose to keep it in the final version of the scale. The Cronbach's $\alpha$ value was 0.75 for the whole sample and 0.74 and 0.74 for the treated and untreated groups, respectively.

\section{Factor analysis}

In the whole sample, the KMO index was 0.79 , confirming the sampling adequacy. The factor analysis and the scree plot indicated a three-factor solution with eigenvalues higher than 1 that explained 58\% of the total variance (Table 2). Factor I was composed of four items on sleep paralysis and hallucinations (questions 11, 12, 13, 14), factor II included eight items on EDS and nighttime sleep (questions $1,2,3,4,5,6,7,15)$ and factor III three items on cataplexy (questions 8, 9, 10). Communalities, which can be interpreted as the proportion of each 
variable variance that can be explained by the factor analysis, were higher than 0.40 (range $=0.43-0.80$; mean 0.58), except for questions 6 and 7 with communalities equal to 0.38 . The item loading values were generally high, ranging from 0.46 to 0.88 . Reliability, expressed by the Cronbach's $\alpha$ value of each factor was satisfactory (range $=0.74-0.85$ ).

\section{Temporal stability}

Among the 70 untreated patients at the first evaluation, eight still untreated patients completed the NSS at a second evaluation after a median duration of 136 days (range: 6-247 days). Among the 105 treated patients at the first evaluation, 21 completed the NSS scale a second time after a median interval of 118 days (range: 25-168 days) (unchanged drugs and dosages). The total score and the item scores were not significantly different between evaluations in the 21 treated patients (NSS total score: $21.57 \pm 8.54$ and $20.71 \pm 8.04$, respectively; $p=0.40$ ) and in the eight drug-free patients $(35.63 \pm 12.44$ and $31.25 \pm 9.19$, respectively; $\mathrm{p}=0.12)$ (Figure 2).

\section{Sensitivity and discriminant validity}

Comparison of the two populations of the independent sample (29 drug-free patients and 105 treated patients) showed that the NSS total score was significantly higher in the untreated than treated group with a ten unit differences between groups $(30.17 \pm 9.57$ versus $22.40 \pm 9.97$, $\mathrm{p}=0.0003$ ) (Figure 2). In the dependent sample (41 patients who were drug-free at baseline and treated at the second evaluation), the NSS total scores also were significantly higher at baseline than after treatment $(34.27 \pm 10.56$ versus $26.00 \pm 10.93, \mathrm{p}<0.0001)$ (Figure 2).

The distributions of the total scores for the two drug-free populations were near the center of the possible range of scores, without a ceiling effect. They ranged from 8 to 50 and from 12 to 53 for the untreated patients in the independent and dependent samples, respectively. Total scores for treated patients in the independent and dependent samples ranged from 2 to 53 and from 1 to 46, respectively (Suppl Figure 1). 
The cut-off value for discriminating treated and drug-free groups, according to the maximum value of the Youden Index, was 24 (sensitivity: 61\%, 95\%CI [52-70]; specificity: $81 \%$ 95\%CI [72-90]).

\section{Convergent validity: Association between NSS scores and clinical or sleep parameters}

The NSS total score significantly and positively correlated with EDS level assessed by the ESS in both treated $(\mathrm{p}<0.0001)$ and drug-free patients $(\mathrm{p}=0.03)$ (Table 3). Similarly, factor II of the NSS that includes eight items mostly relative to EDS, was significantly associated with the ESS score in drug-free and in treated patients ( $\mathrm{p}=0.02$ and $\mathrm{p}<0.0001$, respectively). On the other hand, the NSS total score was negatively correlated with the mean sleep latency on the MSLT in drug-free patients $(\mathrm{r}=-0.41, \mathrm{p}=0.001)$. The NSS total score was positively and significantly correlated with the BDI-II (only in treated patients; $\mathrm{p}<0.0001$ ) and ISI scores, and negatively correlated with the self-reported EQ-5D score in treated and untreated patients (Table 3).

\section{DISCUSSION}

The Narcolepsy Severity Scale (NSS) is a brief instrument that gives a clear quantification of the main narcolepsy symptoms and of their changes following treatment. Its internal consistency, content validity, reproducibility and responsiveness to medication indicate that NSS is a valid, reliable and informative tool for assessing NT1 symptom severity and to detect clinically significant changes upon treatment. The NSS assesses all clinical symptoms of narcolepsy (EDS, cataplexy, hallucinations, sleep paralysis and disturbed nighttime sleep) with a good balance of items on these key symptoms that were selected and validated by sleep experts who took into account also the patients' feedback.

Efficient clinical instruments are needed for assessing the severity of narcolepsy, NT1 subjective symptoms and their consequences. Subjective and objective measures (e.g., ESS, 
MSLT, MWT, Sustained Attention to Response Task and the Psychomotor Vigilance Task) are available to assess EDS or related components and often are used to provide guidance on whether EDS treatment goals are met in patients with $\mathrm{NT} 1^{12,16-19}$. However, to our knowledge, no previous instruments fully evaluated the entire spectrum of NT1 symptoms. NSS is the first patient-reported evaluation of the frequency, severity and consequences of the five classical NT1 symptoms. Moreover, the aim of other tools, such as the Ullanlinna Narcolepsy Scale $\mathrm{e}^{20}$ and the score assessing sleep propensity in active situations developed for narcolepsy $^{21}$, is to discriminate patients with narcolepsy from controls or other hypersomnolent patients. Conversely, the NSS aim is not to diagnose NT1, but rather to quantify the symptom severity following the diagnosis of NT1.

We found that the NSS has good psychometric properties with significant item-total score correlations with adequate internal consistency, except for item 5. However, we chose to keep this item in the NSS because it concerns the refreshing character of daytime sleep episodes, a major narcolepsy feature. ${ }^{1,2}$ The factor analysis indicated a three-factor solution with good reliability, expressed by satisfactory Cronbach's $\alpha$ values. We confirmed that the NSS total score is reproducible with the absence of significant changes in the test-retest evaluation in both treated and untreated patients. However, the total score was slightly decreased in the testretest evaluation, possibly due to NT1 symptom fluctuations when the interval between evaluations was longer. ${ }^{1,2}$ Altogether, the internal consistency, validity and reproducibility analyses gave results for the NSS total score that meet the quality standards for such an instrument.

The aim of the self-reported quantification of the narcolepsy symptom severity in the NSS is to cover all disabilities related to the disease at baseline, but also to monitor the severity changes after treatment. We report significant changes of the NSS total score in the dependent sample before and after medication (median treatment duration of 11 months). Comparison of 
the NSS scores in the independent sample (drug-free and treated patients) also confirmed the lower scores in treated patients with a ten unit differences between groups, without a ceiling effect. A cut-off of 24 showed a good specificity (81\%) in discriminating treated and untreated patients. As the NSS is sensitive to changes in symptom severity, the scale could be potentially useful for the initial and follow-up evaluations to monitor and optimize NT1 management.

Adequate tools to assess narcolepsy severity to improve its clinical management and to help in the development of new therapies are missing. ${ }^{22,23}$ Several subjective and objective tests are currently used to measure various aspects of sleepiness in different sleep disorders, including narcolepsy. ${ }^{11,12,16-19}$ We report here significant correlations between the NSS and the ESS (measurement of daytime sleepiness), and MSLT (sleep latency) scores. The MWT is often proposed for the assessment of the capacity to drive in patients with EDS and of the efficacy of EDS treatments in clinical trials. ${ }^{24-26}$ However this test is infrequently used as a routine measure due to its complexity, required time and cost. Regular monitoring with the NSS after treatment start may be a useful approach to assess the treatment efficacy and to guide treatment changes, such as dose adjustment or drug switching. For this reason, the NSS includes a question on driving and sleepiness at the wheel (item 7). Moreover, the significant correlations between the NSS total score and the BDI-II (depression), ISI (insomnia symptoms) and EQ-5D (quality of life) scores support the convergent validity of the scale. All these findings confirm that the NSS is a relevant tool to evaluate all the main narcolepsy symptoms and their clinical consequences. Moreover, as the NSS is a self-reported questionnaire, the patient becomes an active participant in the assessment/quantification of the main symptomatic complaints and in the treatment decisions and goals.

Our study has some strengths and limitations. We evaluated NSS psychometric properties in a large well-defined population of 175 adult patients with NT1 (untreated or treated), including 
a subgroup with two assessments in both conditions. The study was conducted in a single center, the National Reference Center for Narcolepsy of Montpellier (France) (a tertiary university hospital). Additional research is needed to determine NSS comprehensiveness and applicability to other potentially less severe narcoleptic populations and to validate optimal cut-offs to determine the clinical significance of the complaints and the minimal score change associated with successful treatment. As no other scale or gold standard exists to quantify NT1 severity, no comparison could be made. Many other instruments, such as the ESS, BDIII, ISI, EQ-5D, and objective tests (i.e., MSLT and MWT) are available and were used for comparison in this study. However, we did not include the patient global impression (PGI) or clinical global impression (CGI) ratings. A more formal test-retest evaluation in similar conditions within a 2-week interval between the two administrations of the NSS is recommended. $^{27}$ We also plan to test the NSS in children with NT1; however, younger children could have problems in fully understanding the different questions and the item on driving should be eliminated in a future pediatric version.

In conclusion, we developed a brief self-reported scale to assess NT1 symptom frequency, severity and consequences. The NSS shows good psychometric properties and is sensitive enough to detect changes in symptoms following treatment. Additional studies are needed to further validate this instrument in clinical and research settings, and to better assess the score changes over time following treatment and in clinical trials. If further validated, the NSS may become a clinically relevant tool for improving NT1 management and for providing guidance on whether treatment goals are met.

\section{REFERENCES}


1. Dauvilliers Y, Arnulf I, Mignot E. Narcolepsy with cataplexy. The Lancet. 2007;369:499-511.

2. Scammell TE. Narcolepsy. N Engl J Med. 2015;373:2654-2662.

3. American Academy of Sleep Medicine. International classification of sleep disordersthird edition (ICSD-3). Darien, Illinois; 2014.

4. Dauvilliers Y, Siegel JM, Lopez R, Torontali ZA, Peever JH. Cataplexy - clinical aspects, pathophysiology and management strategy. Nat Rev Neurol. 2014;10:386-395.

5. Roth T, Dauvilliers Y, Mignot E, et al. Disrupted nighttime sleep in narcolepsy. J Clin Sleep Med. 2013;9:955-965.

6. Benca RM. Narcolepsy and excessive daytime sleepiness: diagnostic considerations, epidemiology, and comorbidities. J Clin Psychiatry. 2007;68:5-8.

7. Daniels E, King MA, Smith IE, Shneerson JM. Health-related quality of life in narcolepsy. J Sleep Res. 2001;10:75-81.

8. Dauvilliers Y, Paquereau J, Bastuji H, Drouot X, Weil J-S, Viot-Blanc V. Psychological health in central hypersomnias: the French Harmony study. J Neurol Neurosurg Psychiatry. 2009;80:636-641.

9. Ohayon MM. Narcolepsy is complicated by high medical and psychiatric comorbidities: a comparison with the general population. Sleep Med. 2013;14:488-492.

10. Black J, Reaven NL, Funk SE, et al. The Burden of Narcolepsy Disease (BOND) study: health-care utilization and cost findings. Sleep Med. 2014;15:522-529. 
11. Johns MW. A new method for measuring daytime sleepiness: the Epworth sleepiness scale. SLEEP. 1991;14:540-545.

12. Mitler MM, Gujavarty KS, Browman CP. Maintenance of wakefulness test: a polysomnographic technique for evaluating treatment efficacy in patients with excessive somnolence. Electroencephalogr Clin Neurophysiol. 1982;53:658-661.

13. Beck A, Steer R, Brown G. Beck depression inventory-II. Psychological Corporation. San Antonio, TX; 1996.

14. Bastien $\mathrm{CH}$, Vallières A, Morin CM. Validation of the Insomnia Severity Index as an outcome measure for insomnia research. Sleep Med. 2001;2:297-307.

15. The EuroQol Group. EuroQol-a new facility for the measurement of health-related quality of life. Health Policy. 1990;16:199-208.

16. Carskadon MA, Dement WC, Mitler MM, Roth T, Westbrook PR, Keenan S. Guidelines for the multiple sleep latency test (MSLT): a standard measure of sleepiness. SLEEP. 1986;9:519-524.

17. Dinges DF, Powell JW. Microcomputer analyses of performance on a portable, simple visual RT task during sustained operations. Behav Res Methods Instrum Comput. $1985 ; 17: 652-655$.

18. Manly T, Robertson IH, Galloway M, Hawkins K. The absent mind: further investigations of sustained attention to response. Neuropsychologia. 1999;37:661-670.

19. Van der Heide A, Van Schie MK, Lammers GJ, et al. Comparing Treatment Effect Measurements in Narcolepsy: The Sustained Attention to Response Task, Epworth Sleepiness Scale and Maintenance of Wakefulness Test. SLEEP. 2015;38:1051-1058. 
20. Hublin C, Kaprio J, Partinen M, Koskenvuo M, Heikkilä K. The Ullanlinna Narcolepsy Scale: validation of a measure of symptoms in the narcoleptic syndrome. J Sleep Res. 1994;3:52-59.

21. Sturzenegger C, Bassetti CL. The clinical spectrum of narcolepsy with cataplexy: a reappraisal. J Sleep Res. 2004;13:395-406.

22. Thorpy MJ, Dauvilliers Y. Clinical and practical considerations in the pharmacologic management of narcolepsy. Sleep Med. 2015;16:9-18.

23. Barateau L, Lopez R, Dauvilliers Y. Treatment Options for Narcolepsy. CNS Drugs. 2016;30:369-379.

24. US Modafinil in Narcolepsy Multi- center Study Group. Randomized trial of modafinil for the treatment of pathological somnolence in narcolepsy. Ann Neurol. 1998;43:88-97.

25. Black J, Houghton WC, Xyrem International Study Group. Sodium oxybate improves excessive daytime sleepiness in narcolepsy. SLEEP. 2006;29:939-946.

26. Dauvilliers Y, Bassetti C, Lammers GJ, et al. Pitolisant versus placebo or modafinil in patients with narcolepsy: a double-blind, randomised trial. Lancet Neurol. 2013;12:1068-1075.

27. Terwee CB, Bot SD, de Boer MR, et al. Quality criteria were proposed for measurement properties of health status questionnaires. J Clin Epidemiol. 2007;60:34-42. 
Table 1. Socio-demographic, clinical and polysomnographic characteristics of drug-free and treated patients with narcolepsy in the independent and dependent samples

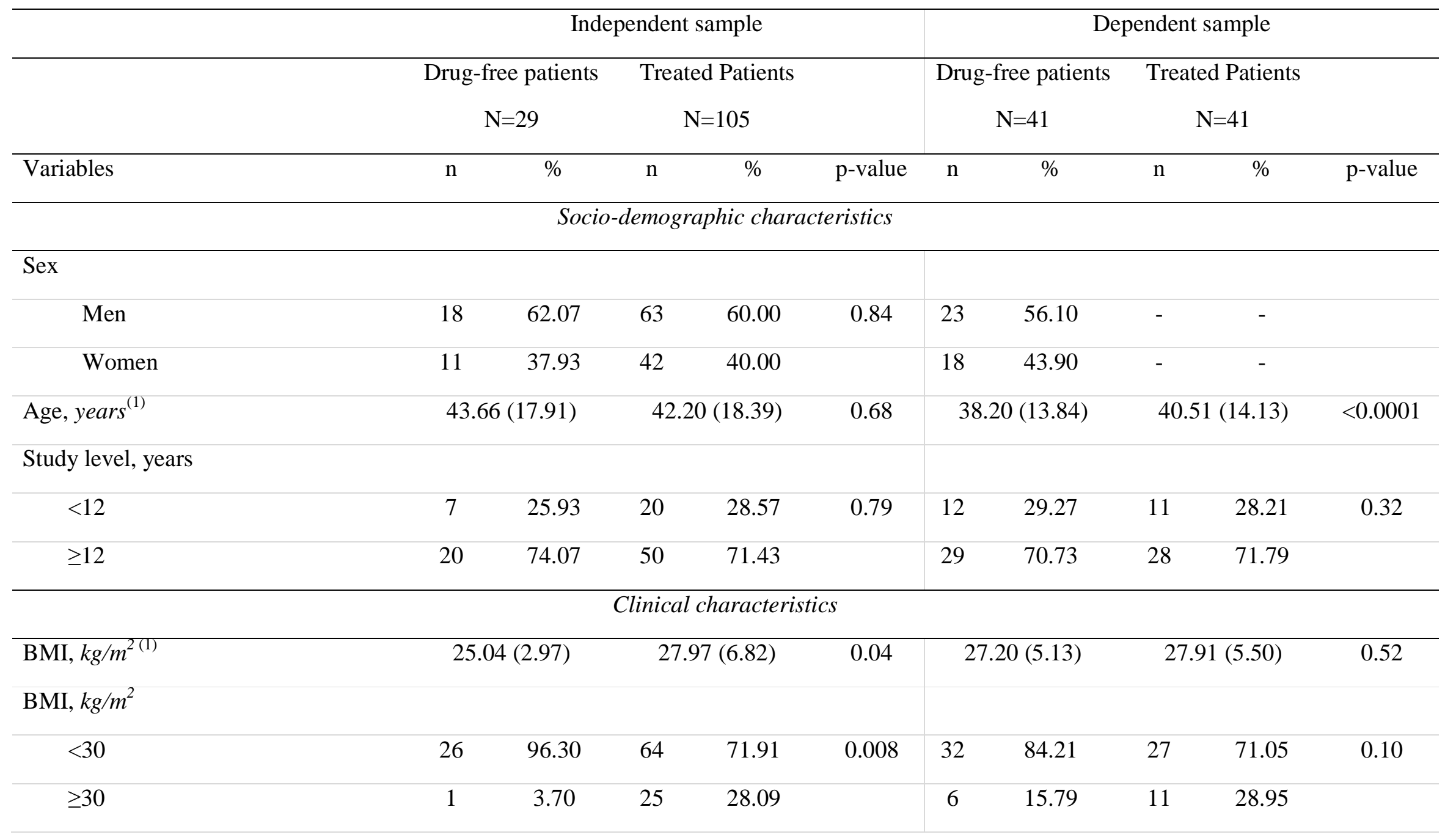




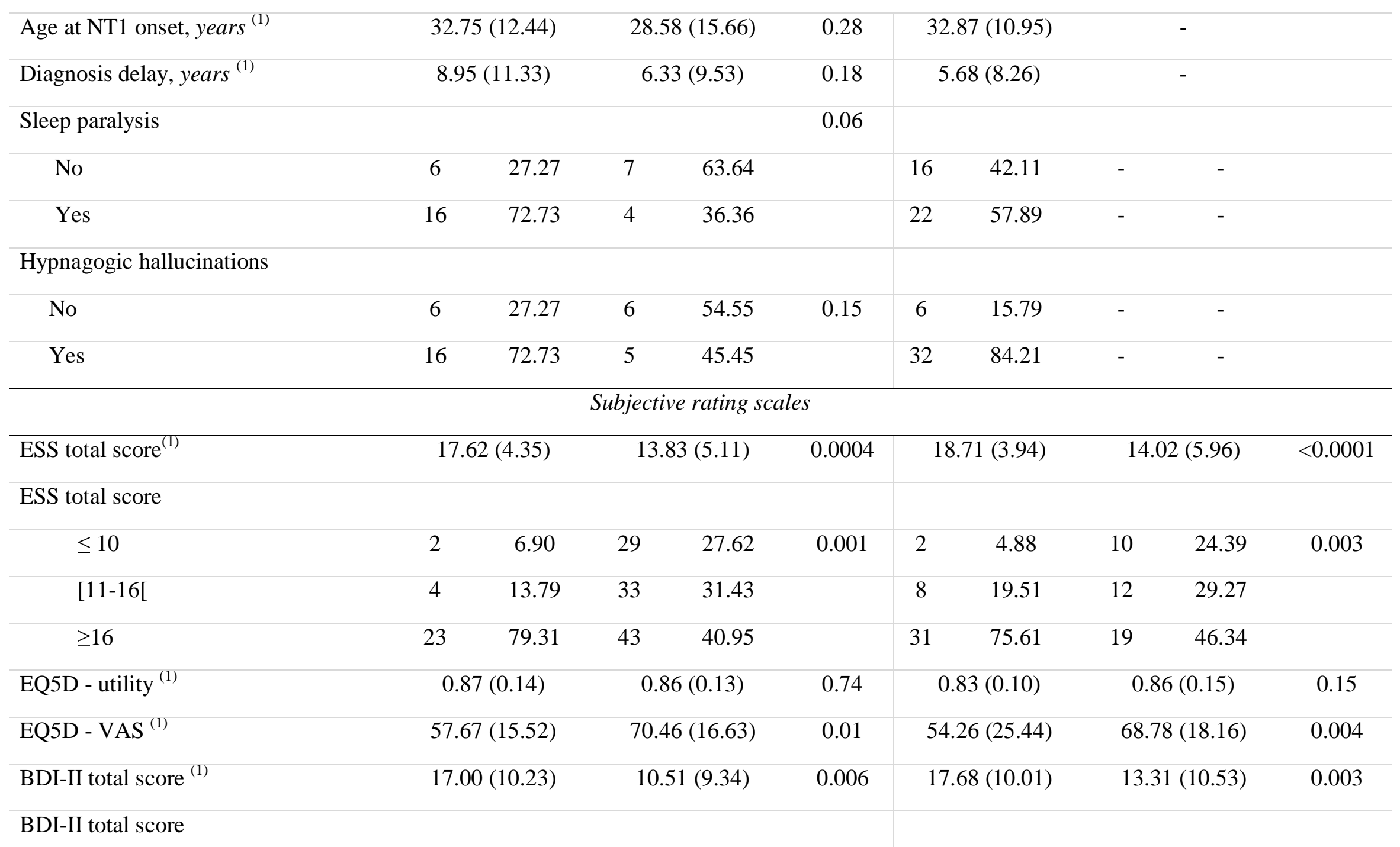




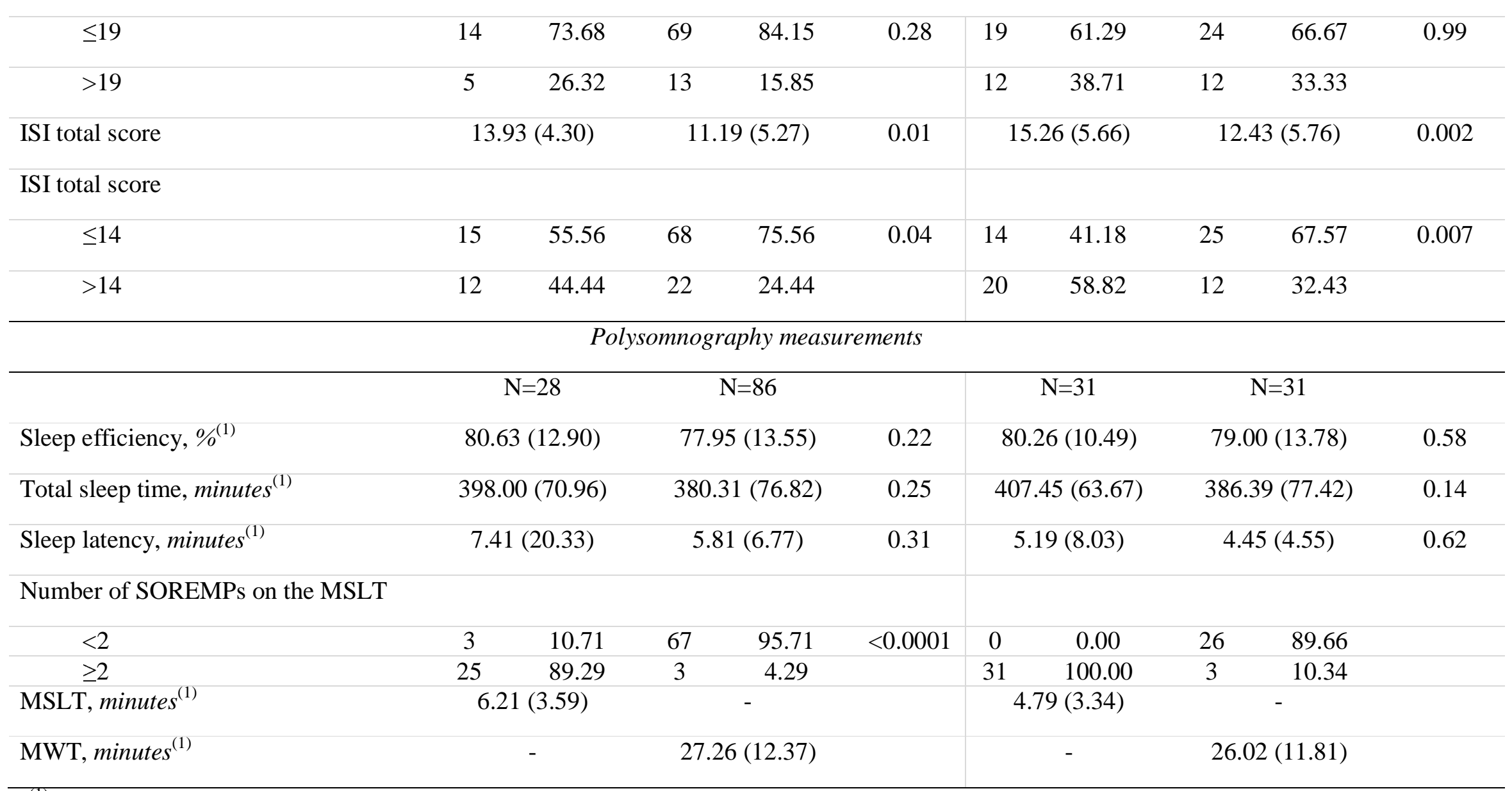

\footnotetext{
${ }^{(1)}$ Continuous variables are expressed as mean (SD)
} 
Table 2. Narcolepsy Severity Scale items - Factor analysis

\begin{tabular}{|c|c|c|c|}
\hline & \multicolumn{3}{|c|}{ Whole population: 175 patients } \\
\hline \multirow[b]{2}{*}{ Questions } & Communalities & \multicolumn{2}{|c|}{ Factors } \\
\hline & $\mathrm{I}$ & II & III \\
\hline 1 & 0.43 & 0.55 & \\
\hline 2 & 0.51 & 0.68 & \\
\hline 3 & 0.56 & 0.68 & \\
\hline 4 & 0.61 & 0.71 & \\
\hline 5 & 0.41 & 0.58 & \\
\hline 6 & 0.38 & 0.50 & \\
\hline 7 & 0.38 & 0.59 & \\
\hline 8 & 0.73 & & 0.81 \\
\hline 9 & 0.65 & & 0.80 \\
\hline 10 & 0.62 & & 0.62 \\
\hline 11 & 0.67 & & \\
\hline 12 & 0.76 & & \\
\hline 13 & 0.79 & & \\
\hline 14 & 0.80 & & \\
\hline 15 & 0.43 & 0.46 & \\
\hline Cronbach's $\alpha$ & 0.85 & 0.77 & 0.74 \\
\hline KMO measure of sampling adequacy & 0 . & & \\
\hline $\begin{array}{l}\text { Percentage of cumulative variance } \\
\text { explained }\end{array}$ & 0. & & \\
\hline
\end{tabular}


Table 3. Association between NSS total score, rating scale scores and sleep latency in drug-free and treated patients with narcolepsy

\begin{tabular}{|c|c|c|c|c|}
\hline \multirow[b]{3}{*}{ Measurements } & \multirow{2}{*}{\multicolumn{2}{|c|}{$\begin{array}{c}\text { Drug-free patients }(\mathrm{N}=70) \\
\text { NSS - Total Score }\end{array}$}} & \multirow{2}{*}{\multicolumn{2}{|c|}{$\begin{array}{l}\text { Treated patients }(\mathrm{N}=105) \\
\text { NSS - Total Score }\end{array}$}} \\
\hline & & & & \\
\hline & $\begin{array}{l}\mathrm{n} ; \text { mean }(\mathrm{SD}) \text { or } \\
\text { correlation coefficient }\end{array}$ & p-value & $\begin{array}{c}\mathrm{n} ; \text { mean }(\mathrm{SD}) \text { or } \\
\text { correlation coefficient }\end{array}$ & p-value \\
\hline ESS total score & & 0.03 & & $<0.0001$ \\
\hline$\leq 10$ & $4 ; 22.50(7.59)$ & & $29 ; 14.59(7.54)$ & \\
\hline$[11-16[$ & $12 ; 28.58(10.36)$ & & $33 ; 21.48(6.37)$ & \\
\hline$\geq 16$ & $54 ; 34.20(9.92)$ & & $43 ; 28.37$ (9.89) & \\
\hline ESS total score & $70 ; 0.46$ & $<0.0001$ & $105 ; 0.61$ & $<0.0001$ \\
\hline $\begin{array}{l}\text { Mean sleep latency on } \\
\text { MWT }\end{array}$ & - & & $24 ;-0.32$ & 0.13 \\
\hline $\begin{array}{l}\text { Mean sleep latency on } \\
\text { MSLT }\end{array}$ & $59 ;-0.41$ & 0.001 & - & \\
\hline BDI-II total score & & 0.13 & & 0.003 \\
\hline$\leq 19$ & $33 ; 31.82(8.97)$ & & $69 ; 20.68(8.92)$ & \\
\hline$>19$ & $17 ; 36.41(11.58)$ & & $13 ; 30.77(10.74)$ & \\
\hline BDI-II total score & $50 ; 0.41$ & 0.003 & $82 ; 0.45$ & $<0.0001$ \\
\hline \multicolumn{5}{|l|}{ ISI total score } \\
\hline$\leq 14$ & $29 ; 28.55(9.63)$ & 0.03 & $68 ; 19.32(7.97)$ & $<0.0001$ \\
\hline$>14$ & $32 ; 34.84(8.57)$ & & $22 ; 32.32(7.77)$ & \\
\hline ISI total score & $61 ; 0.46$ & 0.0002 & $90 ; 0.64$ & $<0.0001$ \\
\hline EQ-5D - Utility & $33 ;-0.37$ & 0.03 & $80 ;-0.37$ & 0.0007 \\
\hline EQ-5D - VAS & $31 ;-0.57$ & 0.0009 & $81 ;-0.34$ & 0.002 \\
\hline
\end{tabular}




\section{Figure 1: Study flow chart}

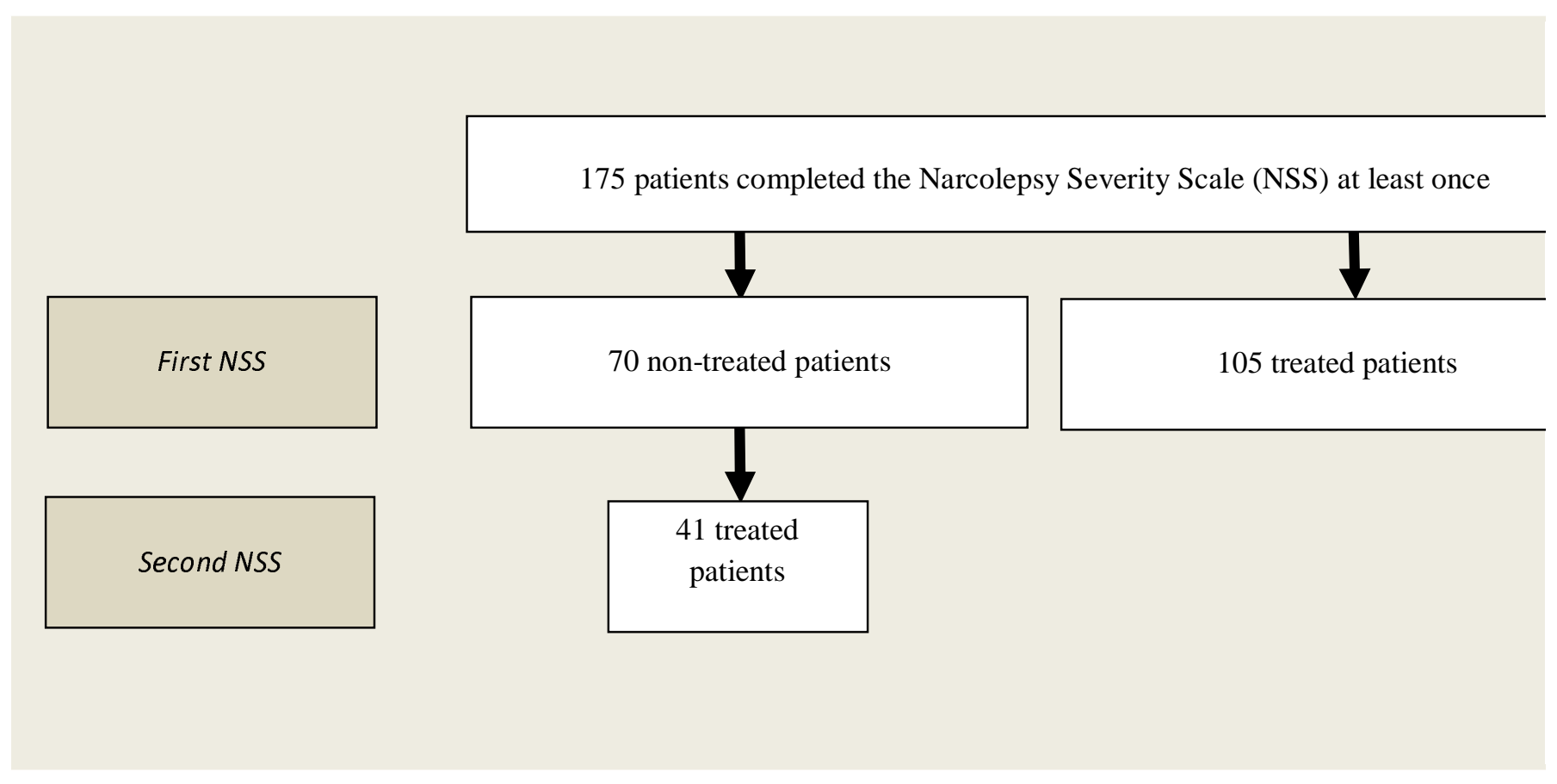


Figure 2. NSS total score in the different samples.

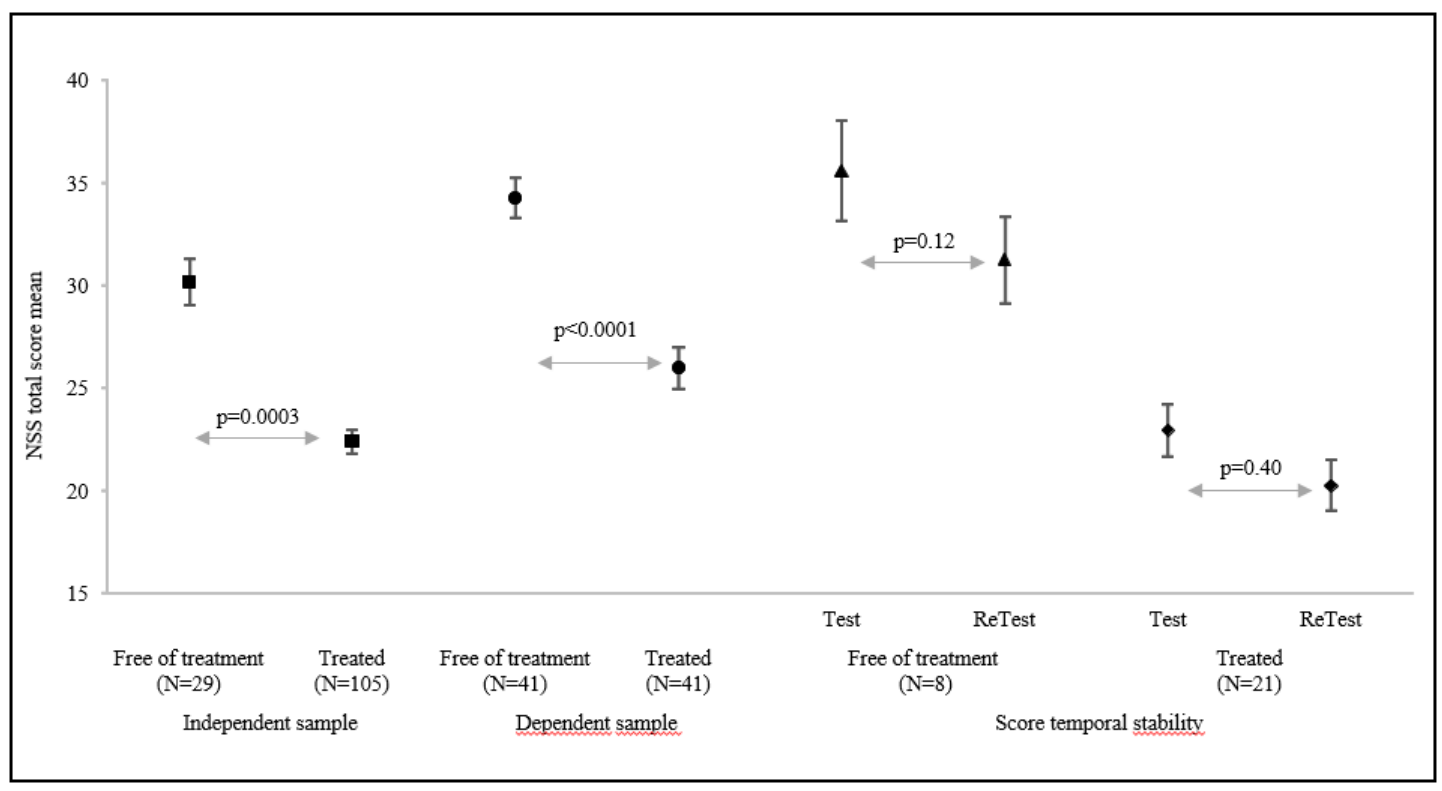

Bars indicate the $95 \%$ mean confidence interval. 
Suppl Figure 1. NSS total score distribution in drug-free and treated patients with NT1 A. Dependent sample (41 patients evaluated twice: first in drug-free and then in treated condition), B. Independent sample (29 patients in drug-free and 105 patients in treated condition).

A

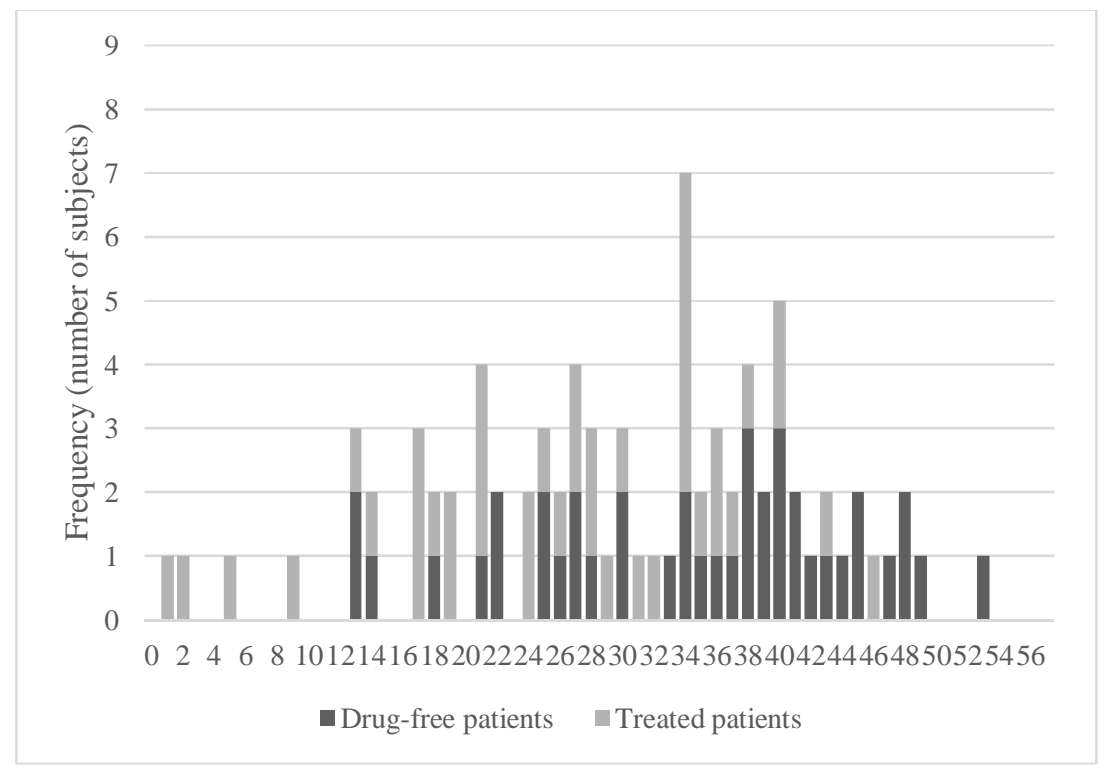

B

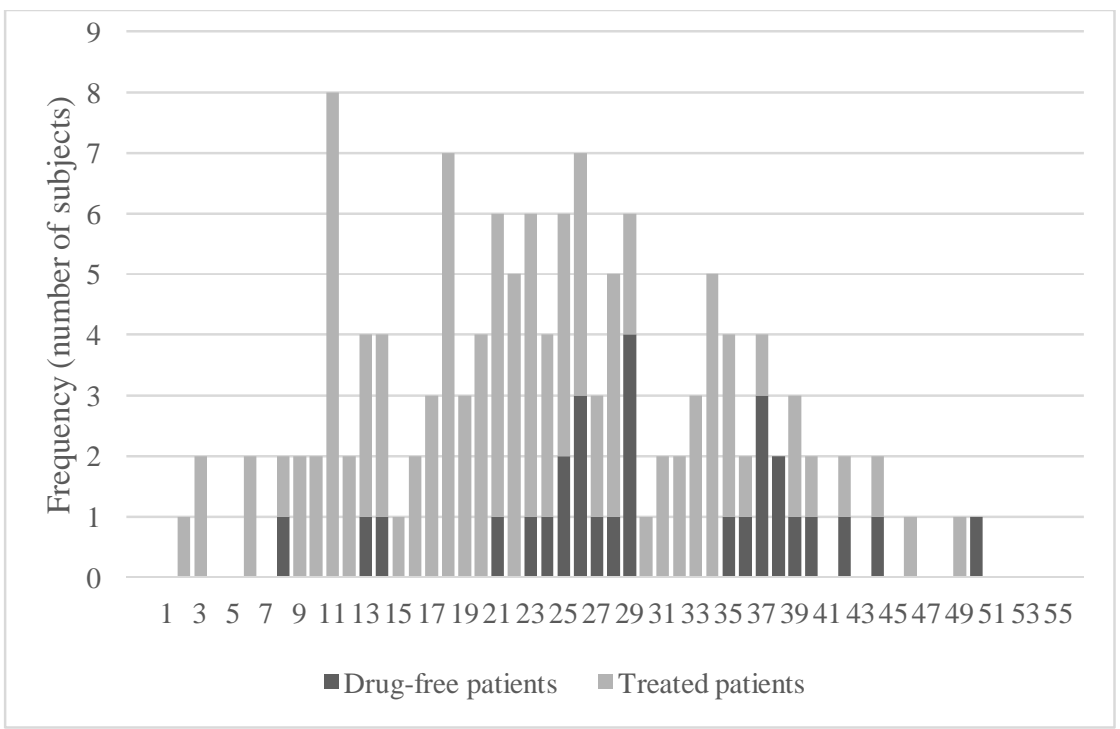

